\title{
Correspondence
}

\section{Possible association of herpes simplex type I reactivation with epidural morphine administration}

To the Editor:

A recent article' described the development of herpes zoster 14 days following an axillary nerve block for surgery following trauma. The herpetic lesion was in the area of infiltration of the regional block. The author stated that "this was the first reported case of herpes zoster chronologically related to an axillary block." Herpes zoster virus (HZV) infection has previously been reported following spinal anaesthesia. ${ }^{2}$

The report is of interest to us as we have noticed an apparent increased incidence of recrudescence of another herpes virus, herpes simplex type I (HSV-I, herpes labialis, "cold sore") when epidural anaesthesia for Caesarean section has been followed by the use of epidural morphine for postoperative analgesia. To date, we have been hesitant to draw attention to this as there are many known non-specific triggers of herpes recrudescence. These include trauma, hormonal changes, sunlight and stress.

A prospective pilot study was done at the Grace Hospital in 1984 to examine the incidence of HSV-I recrudescence in post-partum patients. The Grace Hospital is a 122-bed obstetric hospital, which has over 7,500 deliveries per year, approximately 22 per cent by Caesarean section. All patients who had a Caesarean section during a three-month period (total 556) received a questionnaire to elucidate the incidence of post-partum HSV-I recrudescence. Twenty of the patients were observed to develop a "cold sore" in hospital or responded affirmatively to the questionnaire after discharge (incidence 3.59 per cent). Eighteen of the 20 patients had had epidural anaesthesia followed by epidural morphine for postoperative analgesia. Two patients had general anaesthesia. As well, a random sample of 255 patients who delivered vaginally received a similar questionnaire, which was then followed up by a telephone call three to four weeks after discharge, to discover the incidence of recnudescence of HSV-I in this population. Only five (1.96 per cent) of these patients developed cold sores. None of these five patients had received epidural analgesia during labour. Thirty-five per cent of patients in labour received epidural analgesia during this study.
The results of this pilot study were not statistically significant but they have demonstrated the need for a larger prospective study. A striking aspect of the pilot study has been the severity of the recrudescence; the patients stating that the lesions were more extensive and severe than previously experienced (e.g., extending up onto the nose and eyelid). Typically, the lesions appeared on the second or third postoperative day and many patients felt that the itching experienced from the epidural morphine had been a triggering factor. Some patients who have received epidural morphine on a second occasion (for the birth of another child) developed herpes labialis again the second time. A retrospective study of over 3500 patients who had received epidural morphine following epidural anaesthesia for Caesarean section at the Grace Hospital again revealed an incidence of HSV-I recrudescence of approximately four per cent.

There has been one previous report of herpes simplex following spinal morphine which was used for the treatment of enuresis in children. One day after the procedure, five children (of 55 treated) developed herpes simplex on the upper lip and/or nose. ${ }^{3}$

A large study encompassing 6000 patients has been planned in an endeavour to more clearly define this problem. It is important to remember that association of HSV-I with epidural anaesthesia and epidural morphine does not imply a causal relationship. This apparent association has, however, been discussed at several meetings and it has been brought to our attention by a hospital administrator that he had watched a television program on which a physician stated that "epidural morphine causes recurrence of HSV-I." To date, it has not been proven that either the epidural morphine or the local anaesthetic is the responsible factor, or that even a definite association exists. We feel, therefore, that it is important to not only make our colleagues aware of the possible association but also to caution them that it is necessary to have more complete data on this interesting possibility before discussing it in the public forum.

\author{
M. Joanne Douglas MD \\ G.H. McMorland $M B$ \\ Department of Anaesthesia \\ Grace Hospital and \\ University of British Columbia \\ Vancouver, British Columbia \\ V6H 3V5
}




\section{REFERENCES}

1 Percival NJ. Shingles following axillary nerve block. A case report. J Hand Surg 1986; 11 : 115-6.

2 Vandam $\boldsymbol{L D}$. Complications of spinal and epidural anesthesia. Complications in anesthesiology. Orkin FK, Cooperman LH (Eds). Philadelphia, JB Lippincott 1983; $75-105$

3 Cardan E. Herpes simplex after spinal morphine. Anaesthesia 1984; 39: 1031

\section{Preservative in fentanyl preparations}

To the Editor:

We read with interest the Letter to the Editor by Fukuda and Dohi concerning possible anaphylactic reactions to fentanyl' (Fentanyl-Janssen, Sublimaze).

For the sake of clarity, we wish to point out that only fentanyl vials contain preservatives, the ampoules do not. In most countries, only the ampoules are available. The vials contain methylparasept $0.5 \mathrm{mg} \cdot \mathrm{ml}^{-1}$ and propylparasept $0.05 \mathrm{mg} \cdot \mathrm{ml}^{-1}$.

Another usage area in which this difference could be important is epidural and/or intrathecal analgesia, for which it is advised that only the ampoule-packed fentanyl should be used.

Paul Geerts, DVM

Product Manager CNS-Products

Janssen Pharmaceutica

Turnhoutseweg, 30

B-2340 Beerse

Belgium

\section{REFERENCE}

1 Fukada $T$, Dohi $S$. Anaphylactic reaction to fentanyl or preservative. Can Anaesth Soc J 1986; 33: 826-7.

\section{Use of fiberoptic bronchoscope to assist placement of a Fogarty catheter as a bronchial blocker}

To the Editor:

Although most would agree that tracheal intubation with a double lumen tube is the preferred method for single lung ventilation during thoracic anaesthesia, ${ }^{\prime}$ technical problems occasionally make this procedure difficult. Alternative methods include the passage of an endobronchial blocker such as a Fogarty catheter (American Edwards Laboratories), alongside a single lumen endotracheal

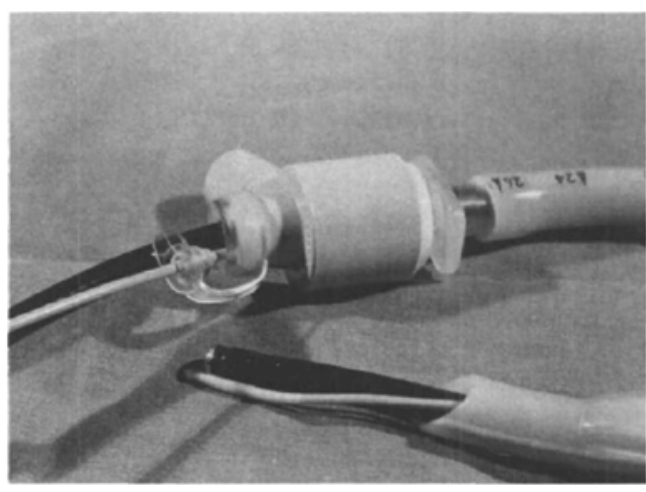

FIGURE 1 Introduction of Fogarty catheter.

tube, through the larynx and trachea, and past the tip of the tube into one of the mainstem bronchi. ${ }^{2}$ This latter technique may also be impractical if airway anatomy makes standard laryngoscopy impossible.

Our patient, a 62-year-old male, presented for mediastinoscopy after routine chest films revealed a lesion in the right upper lobe. After induction of anaesthesia with thiopentone and succiriylcholine, the parient was manually ventilated with bag and mask but several attempts at orotracheal intubation were unsuccess[u]. Spontaneous ventilation was allowed to resume and a blind nasal intubation was performed with difficulty. The mediastinoscopy revealed no evidence of inoperability. In retrospect, the inability to intubate orally was likely secondary to poor neck mobility, limited mouth opening, and a prominent maxilla.

The patient was scheduled for right pneumonectomy. Premedication was with intramuscular morphine and glycopyrrolate and followed by intravenous fentanyl and droperidol. Nebulized four per cent lidocaine provided pharyngeal, laryngeal, tracheal and carinal anaesthesia. Oral fiberoptic intubation with $9.0 \mathrm{~mm}$ cuffed endotracheal tube was performed easily. With the bronchoscope in situ, a 6.0 Fogarty catheter, with the stylet in place, was passed through the clear cap of a fiberoptic bronchoscope adaptor (Portex; Markham, Ontario) (Figure 1), down the lumen of the endotracheal tube, and guided into the right mainstem bronchus. The bronchoscope and Fogarty stylet were removed, and the cap sealed.

Inflation of the Fogarty balloon, manual ventilation, and auscultation of the right chest confirmed the blocker's efficacy. Anaesthesia was then induced and the surgical procedure commenced (Figure 2).

After the right chest was open, the Fogarty balloon was 